\title{
Prevalência de transtornos mentais comuns e fatores associados em uma população assistida por equipes do Programa Saúde da Família
}

\author{
Prevalence of common mental disorders in the population \\ attended by the Family Health Program \\ Juliana Kelly Pinto Moreira', Marina Bandeira', Clareci Silva Cardoso', João Domingos Scalon²
}

\section{RESUMO}

Objetivo: Avaliar a prevalência de casos suspeitos de transtornos mentais comuns em uma população assistida por uma equipe do Programa Saúde da Família e investigar os fatores associados à ocorrência dessa morbidade. Métodos: Pesquisa de levantamento, de corte transversal. A amostra, composta por 277 sujeitos, foi obtida por meio do método de amostragem aleatória do tipo sistemática. Para a coleta de dados, foram aplicados o Self Report Questionnaire (SRQ-20) e um questionário sociodemográfico. Resultados: A prevalência global de casos suspeitos de transtornos mentais comuns na população alvo foi de $43,70 \%$. O sexo feminino apresentou uma taxa de prevalência significativamente mais elevada (48,37\%), quando comparado ao sexo masculino (34,41\%). A variável "renda familiar" apresentou uma relação inversa com a prevalência de transtornos mentais comuns. A análise de regressão logística indicou que apenas as variáveis "uso de medicamento" e "renda familiar" estavam associadas significativamente à suspeita de casos de transtornos mentais comuns. Conclusões: Os resultados apontaram uma elevada prevalência de transtornos mentais comuns nessa população alvo, comparativamente aos dados de outros estudos semelhantes, indicando a necessidade de um cuidado especial por parte da Atenção Primária à Saúde, em particular para as famílias caracterizadas pelos fatores associados ao transtornos mentais comuns apontados nesta investigação.

\begin{abstract}
Objective: Investigate the prevalence of suspected cases of common mental disorders in a population assisted by one professional team of the Brazilian Family Health Program as well as to evaluate its associated factors. Methods: A cross-sectional survey study was carried out using a randomized systematic sampling procedure for selecting 277 subjects from the target population. For data collection, two instruments were administered to the sample, the Self Report Questionnaire (SRQ-20) as well as a sociodemographic questionnaire. Results: The global prevalence of cases suspected of presenting common mental disorders in the target population was of $43.70 \%$. Females had a significantly higher prevalence rate (48.37\%), when compared to males
\end{abstract}

1 Universidade Federal de São João Del-Rei (UFSJ).

2 Universidade Federal de Lavras (UFLA).

30/6/2011

Endereço para correspondência: Marina Bandeira Departamento de Psicologia, Universidade Federal de São João Del-Rei, Praça Dom Helvécio, 74 - Fábricas - 336301-160 - São João del Rei, MG E-mail: bandeira@ufsj.edu.br 


\section{Keywords}

Common mental disorders, Family Health Program, Sistema Básico de Saúde, Primary Health Care Services.
(34.41\%). The variable "family income" had an inverse relation to the common mental disorders prevalence. The logistic regression analysis indicated that only two variables - "use of medication" and "family income" - were significantly associated to common mental disorders. Conclusions: The results indicated a higher prevalence of common mental disorders in this target-population, compared to data from other similar studies, indicating the need for a special attention to be rendered by the primary health care system to detect and treat those cases in families attended by the Brazilian Family Health Program, specially those characterized by the associated factors detected.

\section{INTRODUÇÃO}

A Organização Mundial da Saúde (OMS), em suas dez recomendações para a saúde mental no mundo, apontou a relevância da atuação da Atenção Primária à Saúde (APS) para a promoção de saúde mental nas comunidades. Dentre essas recomendações é dado destaque à função das equipes de saúde básica no desenvolvimento de ações visando ao rastreamento, encaminhamento e monitoramento dos usuários que possuem transtornos mentais'. No contexto brasileiro, o grande potencial para o desenvolvimento dessas ações é o Programa Saúde da Família (PSF), tendo em vista que esse programa foi elaborado como forma de estruturar os serviços da APS no país e servir como primeiro contato da população com os serviços de saúde pública.

Pesquisas têm sido realizadas, em âmbito nacional e internacional, visando analisar a ocorrência de transtornos mentais comuns (TMC) na população. O conceito de TMC foi proposto por Goldberg \& Huxley e se refere a casos que apresentam sintomas não psicóticos, como insônia, fadiga, sintomas depressivos, irritabilidade, esquecimento, dificuldade de concentração e queixas somáticas, que produzem incapacidade funcional das pessoas, mas que não preenchem os requisitos para os diagnósticos do DSM-IV².

Em estudos internacionais, a prevalência de TMC tem variado de 24,6\% a 45,3\%33. Essas pesquisas indicaram a associação entre o baixo nível socioeconômico e a baixa escolaridade ao risco de ocorrência de TMC. No cenário brasileiro, as pesquisas sobre TMC, realizadas com amostras de municípios brasileiros, apresentaram taxas de prevalência que variaram de $17 \%$ a $35 \%$ e confirmaram os fatores associados verificados nos estudos internacionais ${ }^{2,7,8}$.

Poucos foram os estudos realizados no contexto brasileiro, visando investigar a prevalência de TMC especificamente em populações assistidas pelo PSF. As taxas de prevalência de TMC encontradas nesses estudos variaram de 24,6 a 38\%. A maior parte dessas pesquisas encontrou maior risco de TMC para o sexo feminino ${ }^{9-13}$ e em pessoas com maior idade ${ }^{9-13}$. Foi verificado também um risco maior entre as pessoas que apresentavam menor nível de escolaridade e menor renda-13.

Considerando a relevância dessa temática para a organização e planejamento dos serviços de saúde mental e a ca- rência de estudos sobre tal questão, o objetivo dessa pesquisa foi avaliar a prevalência de TMC em uma população assistida pelo PSF em uma cidade de porte médio de Minas Gerais.

\section{MÉTODO}

\section{Amostra}

A amostra foi composta por 277 sujeitos, selecionados aleatoriamente da população alvo assistida pelo PSF-Tejuco (Equipes 0801, 0802 e 0803) do município de São João Del-Rei (MG). Os critérios de inclusão da população alvo foram: ser cadastrado nessas equipes de PSF e ter idade entre 18 a 65 anos. Foram excluídos os usuários dos serviços dos Centro de Atenção Psicossocial (CAPs) e aqueles que apresentavam dificuldade em compreender e responder aos questionários da pesquisa.

A técnica de amostragem utilizada no presente estudo foi do tipo aleatória sistemática que, segundo Contandriopoulos et al. ${ }^{14}$, caracteriza-se pela seleção dos participantes a partir de uma lista da população alvo, utilizando um intervalo fixo denominado fração da amostra. Essa seleção consistiu de três etapas: primeiro, foi selecionada aleatoriamente uma das três equipes do PSF-Tejuco; em segundo lugar, foi selecionada aleatoriamente uma amostra das famílias cadastradas nessa equipe; e, por último, para cada família, foi selecionado aleatoriamente um dos seus membros para ser entrevistado. O tamanho da amostra foi definido a partir de cálculo estatístico para obter um poder de amostra adequado, utilizando a seguinte fórmula ${ }^{15}$ :

$$
n=\frac{2 \times \sigma^{2}}{d^{2}} f(\alpha, \beta)
$$

Foi adotado um poder da amostra (1 - $\beta$ ) =0,99, um nível de significância $a=0,01$, um desvio-padrão $\sigma=2,5$, um semi-intervalo de confiança de $d=1,0$ e um valor calculado em função dos valores de a e $\beta(f(a, \beta)=22,1$. Utilizando esses valores, chegou-se a um tamanho de amostra calculado igual a 276,25 sujeitos. Esse número foi, então, arredondado para 277.

\section{Instrumentos de medida}

Para identificação da ocorrência de TMC, foi utilizada a escala Self Report Questionnaire (SRQ-20). Trata-se de uma escala de 
rastreamento dos TMC, elaborada por Harding et al. ${ }^{16}$ e validada para o contexto brasileiro, primeiramente por Mari e Williams ${ }^{17}$ e, posteriormente, por Gonçalves et al. ${ }^{18}$. Trata-se de uma escala de medida unifatorial, que apresenta propriedades psicométricas adequadas de validade e fidedignidade $^{18}$. Possui 20 questões com alternativas de respostas do tipo "sim" ou "não". Esse instrumento é recomendado pela OMS para estudos de levantamento e em APS, por apresentar facilidade de aplicação e custo reduzido. Foi utilizado também um questionário, previamente testado em estudo-piloto, para avaliar as seguintes variáveis socioeconômicas e demográficas: idade, sexo, cidade de origem, estado civil, escolaridade, número de moradores por domicílio, número de moradores que possuem renda por domicílio, renda familiar, tipo de vínculo empregatício, uso de medicamento e tipo de medicamento.

\section{Procedimento}

As entrevistas foram realizadas individualmente, na residência dos respondentes, segundo sua disponibilidade de horário. Os instrumentos foram aplicados pelos agentes comunitários de saúde (ACS), após um treinamento realizado pela pesquisadora responsável. As perguntas foram lidas e as respostas, anotadas pelos próprios entrevistadores. Os participantes da pesquisa foram informados sobre os objetivos e os procedimentos do estudo, foram assegurados do sigilo das informações fornecidas e assinaram o Termo de Consentimento Livre e Esclarecido, antes do início da entrevista. Essa investigação foi aprovada pelo Comitê de Ética da Universidade Federal de São João Del-Rei (UFSJ) sob o no 014-a/2010/CEPES e pelas autoridades responsáveis pelo serviço municipal de saúde.

\section{Análise de dados}

Para o cálculo da prevalência de TMC foi utilizado, como ponto de corte, escores com valores $\geq 7$ na escala SRQ-20, conforme recomendação de Gonçalves et al. ${ }^{18}$. Foi feita uma análise estatística univariada, com cálculos de $x^{2}$, para avaliar as variáveis sociodemográficas citadas anteriormente e sua relação com a ocorrência de TMC. Realizou-se também uma análise de regressão logística para identificar os principais fatores associados ao risco de ocorrência de TMC, na qual a variável dependente foi a ocorrência de TMC e as variáveis independentes foram as que se mostraram significativas na análise univariada, assim como as variáveis que não foram significativas. Os dados foram analisados por meio do software SPSS-PC, versão 13.0 .

\section{RESULTADOS}

\section{Descrição da amostra}

A tabela 1 apresenta os dados sociodemográficos dos sujeitos. A maioria da amostra era do sexo feminino $(66,40 \%)$, com idade média de 39,05 anos e casada (41,20\%).
Tabela 1. Características sociodemográficas da amostra

\begin{tabular}{|c|c|c|}
\hline Variáveis & Especificaçōes & Frequência (\%) \\
\hline \multirow[t]{2}{*}{ Sexo } & Masculino & $93(33,60)$ \\
\hline & Feminino & $184(66,40)$ \\
\hline Idade & Média & $39,05(\mathrm{DP}+13,54)$ \\
\hline \multirow[t]{2}{*}{ Cidade de origem } & São João Del-Rei & $243(87,70)$ \\
\hline & Outros & $34(12,30)$ \\
\hline \multirow[t]{4}{*}{ Estado civil } & Solteiro & $113(40,80)$ \\
\hline & Casado & $114(41,20)$ \\
\hline & Divorciado & $21(7,60)$ \\
\hline & Viúvo & $29(10,50)$ \\
\hline \multirow[t]{4}{*}{ Escolaridade } & Analfabeto & $14(5,10)$ \\
\hline & Ensino Fundamental & $155(56,00)$ \\
\hline & Ensino Médio & $85(30,70)$ \\
\hline & Ensino Superior & $23(8,30)$ \\
\hline Número de moradores por & $1 \mathrm{a} 2$ & $69(24,90)$ \\
\hline \multirow[t]{2}{*}{ domicílio } & 3 a 4 & $144(51,90)$ \\
\hline & 5 ou mais & $64(23,10)$ \\
\hline Número de moradores que & 1 & $121(43,70)$ \\
\hline \multirow[t]{2}{*}{ possuíam renda por domicílio } & 2 & $119(43,00)$ \\
\hline & 3 ou mais & $37(13,30)$ \\
\hline Tipo de vínculo & Formal & $157(56,70)$ \\
\hline \multirow[t]{2}{*}{ empregatício } & Informal & $96(34,70)$ \\
\hline & Formal e informal & $24(8,70)$ \\
\hline \multirow[t]{3}{*}{ Renda familiar } & Menos de um salário-mínimo & $29(10,50)$ \\
\hline & De um a três salários-mínimos & $212(76,50)$ \\
\hline & Mais de três salários-mínimos & $36(13,00)$ \\
\hline \multirow[t]{2}{*}{ Uso de medicamento } & Sim & $98(35,40)$ \\
\hline & Não & $179(64,60)$ \\
\hline \multirow[t]{6}{*}{ Tipo de medicamento } & Sintomas físicos & $70(71,40)$ \\
\hline & Ansiolíticos & $3(3,10)$ \\
\hline & Antidepressivo & $10(10,20)$ \\
\hline & Antipsicótico & $4(4,10)$ \\
\hline & Antiepilético & $2(2,00)$ \\
\hline & Sintomas físicos e mentais & $9(3,20)$ \\
\hline
\end{tabular}

Quanto ao nível de escolaridade, a maioria (56\%) dos entrevistados cursou apenas o ensino fundamental e 30,70\% concluíram o ensino médio. No que se refere à renda das famílias, houve um predomínio do valor de um a três salários-mínimos por mês (76,50\%). Quanto ao número de pessoas com renda na família, 43,70\% das famílias possuíam apenas uma pessoa com renda e $43,00 \%$, duas pessoas com renda no domicílio. As famílias eram compostas, em sua maioria, por três ou quatro moradores $(51,90 \%)$, sendo que $24,90 \%$ eram compostas por um ou dois moradores e $23,90 \%$, com mais de cinco moradores. A maioria dos sujeitos não fazia uso de medicamentos (64,60\%). Porém, dentre os que utilizavam algum medicamento, a maioria o fazia para tratar de sintomas físicos (71,40\%), enquanto somente $28,60 \%$ utilizaram psicotrópicos, sendo os mais frequentes os antidepres$\operatorname{sivos}(10,20 \%)$. 


\section{Prevalência e análise univariada}

Os resultados mostraram uma prevalência de 43,70\% (IC95\%: 37,9-49,5\%) de TMC na população investigada. As maiores frequências (70\%) de respostas positivas ("sim") ocorreram para a questão n. 6 da escala ("Sente-se tenso, nervoso ou preocupado?"). Com relação às respostas negativas ("não"), a questão que apresentou maior frequência $(87,40 \%)$ foi a de n. 17 ("Tem tido ideia de acabar com a própria vida?").

A análise estatística univariada (Tabela 2) apontou apenas três variáveis estatisticamente associadas à ocorrência de TMC: sexo, uso de medicamento e renda familiar. O risco de ocorrência de TMC foi significativamente mais elevado para o sexo feminino (48,37\%) do que masculino $\left(x^{2}=4,89 ; p<0,05\right)$. Quanto à variável "renda familiar", houve uma diferença significativa entre as três categorias avaliadas $\left(x^{2}=13,87 ; p<0,001\right)$, com maior ocorrência de TMC para o nível inferior de renda. Observou-se que $58,62 \%$ dos sujeitos com renda familiar abaixo de um salário-mínimo apresentaram indicativo de TMC, 46,23\% dos sujeitos com renda familiar de um a três salários-mínimos por mês apresentaram indicativos de TMC e apenas 16,67\% dos entrevistados com renda familiar acima de três salários mínimos mensais apresentaram indicativo de TMC. A terceira variável significativamente relacionada com a prevalência de TMC, na análise univariada, foi o uso de medicamentos $\left(X^{2}=\right.$ $11,17 ; p<0,001)$. A maior parte dos entrevistados $(57,14 \%)$, que apresentou indicativo de TMC, declarou fazer uso de algum medicamento. Essas três variáveis identificadas como significativas na análise univariada, "sexo", "uso de medicamento" e "renda", foram utilizadas no modelo multivariado de regressão logística, juntamente com as variáveis que não foram significativas, coletadas no questionário sociodemográfico.

\section{Análise multivariada}

A tabela 3 mostra os resultados referentes à análise estatística multivariada de regressão logística. Pode-se observar, nessa tabela, que as variáveis que se mantiveram no modelo final, como fatores associados à prevalência de TMC, foram o uso de medicamento $(O R=2,53 ; p \leq 0,05)$ e a renda familiar $(O R=0,37 ; p \leq 0,05)$. Pessoas com TMC apresentaram uma chance 2,53 vezes maior de fazer uso de medicamentos quando comparadas a pessoas sem TMC. As demais variáveis não se mantiveram no modelo multivariado, provavelmente devido às suas intercorrelações com a primeira variável mencionada anteriormente.
Tabela 2. Frequências e porcentagens de sujeitos com indicativo de transtornos mentais comuns por categoria de variáveis

\begin{tabular}{|c|c|c|}
\hline \multirow[b]{2}{*}{ Categorias } & \multicolumn{2}{|c|}{ Indicativo de TMC } \\
\hline & $\begin{array}{l}\text { Acima do ponto de corte } \\
\text { Frequência }(\%)\end{array}$ & $\begin{array}{l}\text { Abaixo do ponto de corte } \\
\text { Frequência }(\%)\end{array}$ \\
\hline \multicolumn{3}{|l|}{ Sexo } \\
\hline Masculino & $32(34,41)^{*}$ & $61(65,59)^{*}$ \\
\hline Feminino & $89(48,37)^{*}$ & $95(51,63)^{*}$ \\
\hline \multicolumn{3}{|l|}{ Idade } \\
\hline De 18 a 44 anos & $79(43,65)$ & $102(56,35)$ \\
\hline De 45 a 65 anos & $43(44,79)$ & $53(55,21)$ \\
\hline \multicolumn{3}{|l|}{ Estado civil } \\
\hline Solteiro & $55(48,67)$ & $58(51,33)$ \\
\hline Outros & $67(40,85)$ & $97(59,15)$ \\
\hline \multicolumn{3}{|l|}{ Cidade de origem } \\
\hline São João Del-Rei & $106(43,62)$ & $137(56,38)$ \\
\hline Outros & $16(47,06)$ & $18(52,94)$ \\
\hline \multicolumn{3}{|l|}{ Cursou a escola } \\
\hline Sim & $115(43,73)$ & $148(56,27)$ \\
\hline Não & $06(42,86)$ & $08(57,14)$ \\
\hline \multicolumn{3}{|l|}{ Escolaridade } \\
\hline Ensino Fundamental & $70(45,16)$ & $85(54,84)$ \\
\hline Ensino Médio e Superior & $44(41,12)$ & $63(58,88)$ \\
\hline \multicolumn{3}{|c|}{ Número de moradores por domicílio } \\
\hline 1 a 2 & $35(50,71)$ & $34(49,28)$ \\
\hline$\geq 3$ & $87(41,83)$ & $121(58,17)$ \\
\hline $\begin{array}{l}\text { Número de moradores que poss } \\
\text { renda } \\
1\end{array}$ & $58(47,93)$ & $63(52,07)$ \\
\hline$\geq 2$ & $64(41,03)$ & $92(58,97)$ \\
\hline \multicolumn{3}{|l|}{ Tipo de vínculo empregatício } \\
\hline Formal & $81(45,22)$ & $100(55,25)$ \\
\hline Informal & $41(41,67)$ & $55(57,29)$ \\
\hline \multicolumn{3}{|l|}{ Renda familiar } \\
\hline < 1 salário-mínimo & $17(58,62)^{* *}$ & $12(41,38)^{* *}$ \\
\hline$\geq 1$ e $<3$ salários-mínimos & $99(46,23)^{* *}$ & $113(53,30)^{* *}$ \\
\hline$\geq 3$ salários-mínimos & $06(16,67)^{* *}$ & $30(83,33)^{* *}$ \\
\hline \multicolumn{3}{|l|}{ Uso de medicamentos } \\
\hline Sim & $56(57,14)^{* *}$ & $42(42,85)^{* *}$ \\
\hline Não & $65(36,31)^{* *}$ & $114(63,69)^{* *}$ \\
\hline \multicolumn{3}{|l|}{ Tipo de medicamento } \\
\hline Doenças físicas & $38(54,29)$ & $32(45,71)$ \\
\hline Psicotrópicos & $18(64,29)$ & $10(35,71)$ \\
\hline
\end{tabular}

${ }^{*} p \leq 0,05 ;{ }^{* *} p \leq 0,001$

TMC: transtornos mentais comuns.

Tabela 3. Resultados da análise de regressão logística para os fatores associados à prevalência de transtornos mentais comuns e dos índices de odds ratio

\begin{tabular}{lccccccc}
\hline Variáveis & OR & LI-LS (IC55) & Valor $\mathbf{p}$ & $\beta$ & EP & Qui-quadrado & Valor $\mathbf{p}$ \\
\hline Intercepto & - & - & - & $+0,42$ & 0,32 & 1,74 & 0,19 \\
Uso de medicamento & 2,53 & $1,51-4,24$ & 0,0010 & $+0,93$ & 0,26 & 12,40 & 0,0004 \\
Renda familiar & 0,37 & $0,21-0,65$ & 0,0006 & $-0,99$ & 0,28 & 11,95 & 0,0005 \\
\hline
\end{tabular}

OR: odds ratio; LI: limite inferior; LS: limite superior; IC95\%: intervalo de confiança de 95\%; EP: erro padrão. 


\section{DISCUSSÃO}

Os resultados da presente pesquisa indicaram uma elevada taxa de prevalência de TMC na população alvo investigada. A prevalência de TMC foi de $43,70 \%$, que consiste em um valor superior ao apresentado nas pesquisas realizadas com populações alvo semelhantes, ou seja, que fazem parte de Programas Saúde da Família, nas quais a ocorrência de TMC variou de $24 \%$ a $38 \% \%^{9-13,19}$. Comparada aos resultados dos estudos aqui citados, a taxa de prevalência verificada no presente trabalho se aproxima mais do resultado do estudo de Gonçalves et al. ${ }^{13}$, no qual o valor observado foi de $38 \%$. Em outros estudos realizados com a uma população alvo de serviços de atenção primária, diferentes do PSF, verificou-se que a prevalência de TMC foi ainda mais elevada, variando entre $41 \%$ e $55 \%{ }^{20}$. A taxa de TMC observada nessa pesquisa é semelhante ao menor valor obtido nos estudos citados por Andreoli ${ }^{20}$. A alta taxa de prevalência observada talvez se explique pelo baixo nível socioeconômico da população alvo estudada. Dados da literatura apontam para a ocorrência de divergências nas taxas de prevalência dos transtornos mentais, em estudos realizados em diversos contextos ou com diferentes populações alvo ${ }^{21,22}$. Essas diferenças nas taxas de prevalência também podem ser observadas em estudos realizados nos municípios brasileiros, não apenas em populações assistidas pelo PSF.

Os resultados da análise de regressão logística indicaram a presença de dois fatores associados à prevalência de TMC: o uso de medicamento e a renda. Esses resultados indicam, portanto, que a ocorrência de TMC foi mais elevada em indivíduos que faziam uso de medicamentos. No presente estudo, foi constatado que, na maioria dos casos, os medicamentos utilizados se destinavam ao tratamento de sintomas físicos. Esse resultado aponta para a necessidade de monitoramento de pacientes que apresentam problemas de saúde física, verificando-se seu impacto na saúde mental. O efeito da variável "uso de medicamento" não pode ser comparado com outros estudos, pois essa não foi investigada nas demais pesquisas. No entanto, os resultados encontrados para a variável uso de medicamentos sugerem uma possível associação entre a utilização de fármacos para tratamento de sintomas físicos e morbidades psiquiátricas. Essa associação foi investigada na literatura, como, por exemplo, no estudo de Benseñor et al..23, no qual foram encontradas morbidades psiquiátricas em pacientes que utilizavam medicamentos para tratamento de hipertensão arterial.

A prevalência de TMC também foi associada a uma menor renda familiar, na análise multivariada, corroborando dados existentes na literatura ${ }^{3,5,9-13}$. Essa associação pode ser explicada pelo fato de a baixa renda estar relacionada a fatores que aumentam a probabilidade de ocorrência de problemas psicológicos, como diminuição do poder, eventos de vida estressantes e desempenho insatisfatório de pa- péis sociais, que acarretariam baixa autoestima, ocasionando maior suscetibilidade aos transtornos mentais ${ }^{10,25-27}$. Dados da literatura apontam que fatores ambientais desfavoráveis contribuem para o aumento da taxa de prevalência de TMC, tais como: dificuldades financeiras, internações hospitalares, problemas interpessoais, mudanças de moradia, acesso desigual aos cuidados de saúde, desemprego, problemas de saúde, condições habitacionais inadequadas, ser vítima de violência e criminalidade ${ }^{27}$.

Os resultados obtidos com a presente pesquisa podem ser generalizados para a população alvo previamente definida, uma vez que a amostra estudada foi selecionada aleatoriamente dessa população. Entretanto, sendo um estudo de corte transversal, essa pesquisa apresenta a limitação de não poder assegurar a relação causal entre as variáveis. Estudos longitudinais futuros poderão acompanhar as alterações ocorridas nas características da comunidade, ao longo do tempo e sua relação com a ocorrência de TMC.

\section{CONCLUSÕES}

Os resultados da presente pesquisa permitiram identificar uma alta taxa de prevalência de TMC na população assistida pelo PSF, em uma cidade de médio porte, apontando para a necessidade de atendimento em saúde mental para essas famílias. Os resultados indicam, ainda, a importância de desenvolver, no PSF, ações relacionadas ao rastreamento e encaminhamento de casos que necessitam de atendimento em saúde mental, o que requer um investimento nos aspectos relacionados aos recursos humanos e de treinamento dos agentes comunitários do PSF para esse fim.

Os resultados permitiram ainda identificar dois fatores associados à prevalência de TMC: a utilização de medicamentos e a renda familiar. Esses dados apontam para a necessidade de um maior cuidado com as famílias de menor renda e para os membros dessas famílias que utilizam medicamentos, tendo em vista que esses possuem maiores chances de apresentar TMC. Esses resultados levam à reflexão sobre a complexidade que envolve o processo saúde/ doença dos indivíduos. Como adverte a OMS', a saúde não significa apenas a ausência de doenças e, sim, um estado de bem-estar biopsicossocial. Em função disso, as intervenções em saúde e, particularmente, em saúde mental, para serem efetivas, devem contar com a participação de diversas áreas dos governos, tais como a educação, a assistência social, o saneamento básico e outras mais. O presente estudo apontou, também, a relevância de realizar pesquisas de levantamento, buscando identificar as principais características da população nas quais são implantados os serviços de saúde, de forma a orientar as ações a serem implementadas pelos serviços, em particular das equipes do PSF. 


\section{REFERÊNCIAS}

1. World Health Organization (WHO) [Internet]. The world health report 2001 - Mental Health: New Understanding, New Hope. [cited 2009 Mar 9] 2001. Available from: http://www. who.int/whr/2001/en/

2. Ludermir AB, Melo Filho DA. Condições de vida e estrutura ocupacional associadas a transtornos mentais comuns. Rev Saúde Pública. 2002;36(3):213-21.

3. Weich S, Lewis G. Poverty, unemployment, and common mental disorders: population based cohort study. BMJ. 1998;317(7151):115-9.

4. Araya R, Fritsch R, Acuña J, Lewis G. Common mental disorders in Santiago, Chile: prevalence and socio-demographic correlates. Br J Psychiatry. 2001;178:228-33.

5. Patel V, Chisholm D, Rabe-Hesketh S, Dias-Saxena F, Andrew G, Mann A. Efficacy and cost-effectiveness of drug and psychological treatments for common mental disorders in general health care in Goa, India: a randomised Controlled Trial. Lancet. 2003;361(9351):33-9.

6. Puertas $G$, Ríos C, Valle $H$. Prevalencia de transtornos mentales comunes em barrios marginales urbanos con población desplazada en Colombia. Rev Panam Salud Publica. 2006;20(5):324-30.

7. Rodrigues-Neto JF, Figueiredo MFS, Faria AAS, Fagundes M. Transtornos mentais comuns e 0 uso de práticas de medicina complementar e alternativa - estudo de base populacional. J Bras Psiq. 2008:57(4):233-9.

8. Marín-Léon L, Oliveira HB, Barros MB, Dalgalarrondo P, Botega NJ. Social inequality and common mental disorders. Rev Bras Psiquiatr. 2007;29(3):250-3.

9. Costa AG, Ludermir AB. Transtornos mentais comuns e apoio social: estudo em comunidade rural da Zona da Mata de Pernambuco, Brasil. Cad Saúde Pública. 2005;21(1):73-9.

10. Maragno L, Goldbaum M, Gianini RJ, Novaes HMD, César CLG. Prevalência de transtornos mentais comuns em populações atendidas pelo Programa Saúde da Família (QUALIS) no Município de São Paulo, Brasil. Cad Saúde Pública. 2006;22(8):1639-48.

11. Fortes $S$, Villano LAB, Lopes (S. Perfil nosológico e prevalência de transtornos mentais comuns em pacientes atendidos em unidades do Programa Saúde da Família (PSF) de Petrópolis, Rio de Janeiro. Rev Bras Psiquiatr. 2008;30(1):32-7.

12. Gianini RJ, Carvalho TC, Anjos RMP, Pinto PLS, Maluf ME, Lanza LB, et al. Prática de rastreamento no cenário do Programa Saúde da Família de Sorocaba (SP). Rev Brasileira Educ Med. 2008;32(1):15-22.

13. Gonçalves DM, Kapczinski F. Transtornos mentais em comunidade atendida pelo Programa Saúde da Família. Cad Saúde Pública. 2008;24(7):1641-50.
14. Contandriopoulos A, Champagne F, Potvin L, Denis J, Boyle P. Saber prepara uma pesquisa - Definição, estrutura e financiamento. São Paulo/Rio de Janeiro: Hucitec/Abrasco; 1994.

15. Cohen J. Statistical power analysis for the behavioral sciences. 2. edition. Hillsdale, NJ: Erlbaum, 1988.

16. Harding TW, De Arango MV, Baltazar J, Climent CE, Ibrahim HHA, Ladrido-Ignacio L. Mental disorders in primary health care: a study of their frequency and diagnosis in four developing countries. Psychol Med. 1980;10:231-41.

17. Mari JJ, Williams P. A validity study of a psychiatric screening questionnaire (SRQ-20) in primary care in the city of São Paulo. Br J Psychiatry; 1986;148:23-6.

18. Gonçalves DM, Stein AT, Kapczinski F. Avaliação de desempenho do Self-Reporting Questionnaire como instrumento de rastreamento psiquiátrico: um estudo comparativo com 0 Structured Clinical Interview for DSM-IV-TR, 2007. Cad Saúde Pública. 2008;24(2):380-90.

19. Bandeira M, Freitas L, Carvalho J. Avaliação da ocorrência de transtornos mentais comuns em usuários do Programa de Saúde da Família. J Bras Psiq. 2007;56(1):41-7.

20. Andreoli SB. Serviços de Saúde Mental no Brasil. In: Mello MF, Mello AAF, Kohn R, orgs. Epidemiologia da saúde mental no Brasil. Porto Alegre: Artmed; 2007.

21. Alem A, Kebede D, Woldesemiat $G$, Jacobsson L, Kullgren $G$. The prevalence and socio-demographic correlates of mental distress in Butajira, Ethiopia. Acta Psychiatrica Scand. 1999;100(48):55.

22. Shidhaye R, Patel V. Association of socio-economic, gender and health factors with common mental disorders in women: a population-based study of 5703 married rural women in India. International Int J Epidemiol. 2010;39(6):1510-21.

23. Benseñor IM, Pereira AC, Tannuri AC, Valeri CM, Akashi D, Fucciolo DQ, et al. Hipertensão arterial sistêmica e morbidade psiquiátrica em ambulatório de hospital terciário. Arq Neuropsiquiatr. 1998;56(3A):406-11.

24. Coutinho ESF, Almeida Filho N, Mari JJ [Internet]. Fatores de risco para morbidade psiquiátrica menor: resultados de um estudo transversal em três áreas urbanas no Brasil. Rev Psiquiatria Clínica. 1999;26(5). [citado em: 2009 Set 25]. Disponível em: http://www.henet. usp.br/ipq/revista/vol26/n5/artigo(246).htm

25. Ludermir AB. Desigualdade de classe e de gênero e saúde mental nas cidades. Physis: Rev Saúde Coletiva. 2008;18(3):451-67.

26. Almeida-Filho N, Mari JJ, Coutinho E, Franca JF, Fernandes J, Andreoli SB, et al. Brazilian multicentric study of psychiatric morbidity. Methodological features and prevalence estimates. Br J Psychiatry. 1997;171:524-9.

27. Lopes CS, Faerstein E, Chor D. Eventos de vida produtores de estresse e transtornos mentais comuns: resultados do Estudo Pró-Saúde. Cad Saúde Pública 2003;19(6):1713-20. 